\title{
Implementation of an antimicrobial stewardship program on the medical-surgical service of a 100-bed community hospital
}

\author{
Donald F Storey ${ }^{1 *+}$, Perry G Pate ${ }^{1 \dagger}$, Autumn $\Pi$ Nguyen $^{2}$ and Fung Chang ${ }^{3}$
}

\begin{abstract}
Background: Antimicrobial stewardship has been promoted as a key strategy for coping with the problems of antimicrobial resistance and Clostridium difficile. Despite the current call for stewardship in community hospitals, including smaller community hospitals, practical examples of stewardship programs are scarce in the reported literature. The purpose of the current report is to describe the implementation of an antimicrobial stewardship program on the medical-surgical service of a 100-bed community hospital employing a core strategy of post-prescriptive audit with intervention and feedback.
\end{abstract}

Methods: For one hour twice weekly, an infectious diseases physician and a clinical pharmacist audited medical records of inpatients receiving systemic antimicrobial therapy and made non-binding, written recommendations that were subsequently scored for implementation. Defined daily doses (DDDs; World Health Organization Center for Drug Statistics Methodology) and acquisition costs per admission and per patient-day were calculated monthly for all administered antimicrobial agents.

Results: The antimicrobial stewardship team (AST) made one or more recommendations for 313 of 367 audits during a 16-month intervention period (September 2009 - December 2010). Physicians implemented recommendation(s) from each of 234 (75\%) audits, including from 85 of 115 for which discontinuation of all antimicrobial therapy was recommended. In comparison to an 8-month baseline period (January 2009 - August 2009), there was a $22 \%$ decrease in defined daily doses per 100 admissions $(P=.006)$ and a $16 \%$ reduction per 1000 patient-days $(P=.013)$. There was a $32 \%$ reduction in antimicrobial acquisition cost per admission $(P=.013)$ and a $25 \%$ acquisition cost reduction per patient-day $(P=.022)$.

Conclusions: An effective antimicrobial stewardship program was implemented with limited resources on the medical-surgical service of a 100-bed community hospital.

Keywords: Antimicrobial stewardship, ASP, Small community hospital

\section{Background}

Hospitals with less than 200 beds accounted for $72 \%$ of American Hospital Association-defined community hospitals in 2008 and $63 \%$ of the acute care facilities reporting to the National Healthcare Safety Network (NHSN) in $2010[1,2]$. Recent reports have demonstrated that large and small hospitals alike comparably share the problem of antimicrobial resistance. An analysis of data

\footnotetext{
* Correspondence: storeydf@gmail.com

${ }^{\dagger}$ Equal contributors

'Dallas ID Associates, Dallas, Texas, USA

Full list of author information is available at the end of the article
}

from the National Nosocomial Infection Surveillance (NNIS) System demonstrated parallel increases in antimicrobial resistance in Staphylococcus aureus blood stream infections, Escherichia coli urinary tract infections and Pseudomonas aeruginosa pneumonias within large and small acute-care facilities between the periods of 1990-1994 and 2000-2004 [3]. In addition, intensive care units of small and large hospitals reporting to NHSN were shown to have comparable proportions of device-associated infections with multidrug-resistant Klebsiella pneumoniae and E. coli [4]. Similarly, the proportion of Acinetobacter baumannii that was multidrug- 
resistant was comparable or greater in smaller facilities compared to larger, tertiary facilities. Furthermore, smaller bed size was independently associated with a higher rate of incident Clostridium difficile infection (CDI) cases reported among 210 Ohio acute care hospitals during 2006 [5].

Antimicrobial stewardship has been promoted for all hospitals to help cope with the challenges of CDI and emerging resistance to antibiotics [6-8]. In 2007, the Infectious Diseases Society of America (IDSA) and the Society for Healthcare Epidemiology of America (SHEA) published revised guidelines for developing institutional programs to enhance antimicrobial stewardship [9]. Several recent surveys have suggested antimicrobial stewardship programs (ASPs) may be more prevalent than appreciated from a review of the medical literature $[10,11]$. Nevertheless, a 2011 report of a survey of infectious diseases (ID) specialists of the IDSA Emerging Infections Network concludes that "small community hospitals still represent the 'frontier' for new stewardship programs" and that they are the hospitals "least likely to have ASPs, the least likely to provide compensation to physicians, and the least likely to believe that any outcomes data might convince administrators to support ASPs" [12]. Even so, smaller hospitals may have higher rates of antimicrobial use than those of large academic medical centers [13]. It has been nearly a decade since the only report of an ASP from a community hospital in the United States with less than 200 beds [14]. We report the implementation of a contemporary ASP on the medical-surgical service of a 100-bed community hospital.

\section{Methods}

\section{Program setting}

An ASP was implemented on the medical-surgical service of a full-service 100-bed community hospital located in an ethnically diverse community in metropolitan Dallas, TX. The 43-bed medical-surgical service consisted of a 24-bed medical-surgical floor unit, 11-bed progressive care unit, and 8-bed medical-surgical intensive care unit. There were no inpatient transplant services, specialized oncology, pediatric, psychiatric, or rehabilitation units. Medical subspecialties were broadly represented and included infectious diseases (ID); surgical specialties included cardiothoracic surgery, colorectal surgery, general surgery, neurosurgery and orthopedic surgery. An electronic medical record was available but without computerized physician order entry or electronic progress notes.

Throughout the reported period, a nurse staffed an infection prevention and control program that included surveillance and prevention activities for multidrugresistant organisms, CDI, and device-associated infections. Since 2006 and throughout the reported period, an ID physician served as the medical director of infection control and employee health. Active admission surveillance testing for methicillin-resistant Staphylococcus aureus was performed on high-risk patients for isolation and cohorting purposes. An off-site laboratory performed microbiology services including preparation of an annual facility antibiogram and polymerase chain reaction testing for CDI. (Laboratory testing for CDI was performed on-site using an enzyme-linked immunoassay for $C$. difficile toxin prior to November 2009).

The pharmacy was staffed by 6.4 full-time-equivalent pharmacists, including a pharmacy director and clinical pharmacy supervisor. The pharmacists did not receive specialized training in infectious diseases.

\section{Interventions}

During the baseline period (January 2009 - August 2009), the ID physician medical director of infection control, the clinical pharmacy supervisor and the pharmacy director together formed an Antimicrobial Stewardship Team (AST) and drafted an antimicrobial stewardship policy and program description for approval by the Pharmacy and Therapeutics Committee and Medical Executive Committee. AST members educated the medical staff about the program through presentations at medical staff committee meetings and through a continuing medical education conference. For approximately one hour twice weekly during the intervention period (September 2009 - December 2010), the ID physician and one or the other of the AST pharmacists audited medical records of inpatients on the medical-surgical service that were receiving more than two days of systemic antimicrobial therapy. On occasion, the AST audited other patient records with shorter durations of therapy. The AST members audited records for prescribed antimicrobial agent(s), clinical indication(s), planned treatment duration(s), drug allergies, renal function, pertinent laboratory and radiographic data. Non-binding written recommendations were made and placed in the record using a communication form that did not become part of the permanent medical record. There were no formulary restrictions or preauthorization requirements.

Additional interventions implemented prior to the baseline period included automatic vancomycin doseoptimization and a pneumonia order set. Order sets for treating patients with severe sepsis or, suspected severe sepsis, and a parenteral to oral conversion protocol were implemented during the intervention period. A timeline with ASP milestones and interventions is displayed in Figure 1.

\section{Data collection}

The AST categorized recommendations as follows: to discontinue all agent(s), to de-escalate antimicrobial 

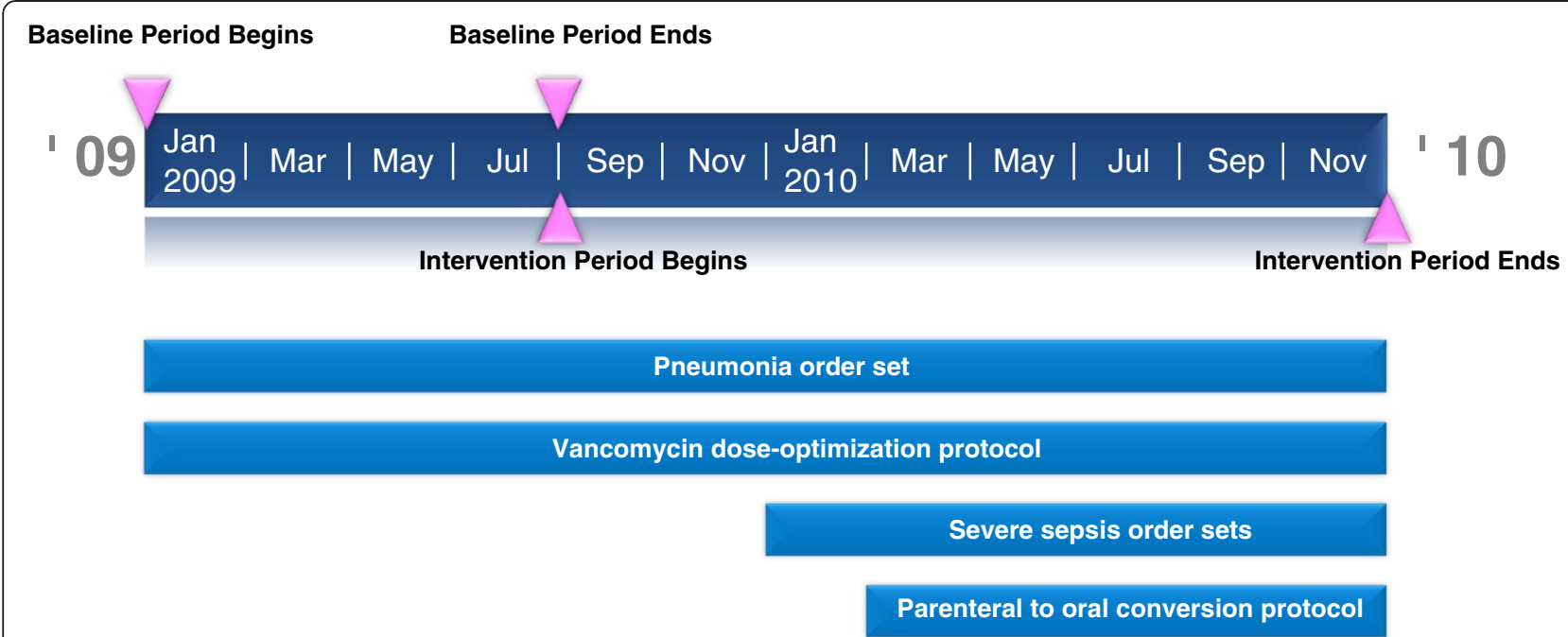

Figure 1 Antimicrobial stewardship program implementation timeline. The timeline depicts antimicrobial stewardship program milestones and interventions. A pneumonia order set and vancomycin dose-optimization protocol were implemented prior to the baseline period. Severe sepsis order sets and a parenteral to oral conversion protocol were implemented during the intervention period. The severe sepsis order sets included choices for initial antibiotic regimen by suspected source of infection and a schedule for continued therapy without automatic stop orders or requirements for physician justification. Individual agents included in the order sets were ampicillin, ampicillin-sulbactam, azithromycin, aztreonam, ceftriaxone, levofloxacin, linezolid, meropenem, piperacillin-tazobactam, rifampin and vancomycin. Antimicrobial agents in the parenteral to oral conversion protocol were fluconazole, levofloxacin, linezolid, metronidazole and voriconazole at the same dose and frequency.

therapy (to discontinue one or more agents and/or substitute an alternate agent(s) with decreased spectrum of activity), to limit duration, to broaden coverage (to add one or more agents and/or substitute alternate agents(s) with increased spectrum of activity), to optimize dose (e.g., for indication, renal function, and/or body weight), to convert route of administration from parenteral to oral and to consult ID. Pharmacist team members followed up recommendations and scored implementation. Data from record review and recommendations were tabulated monthly.

Admissions, patient-days (excluding outpatient-days), Medicare Case Mix Index, and characteristics of patients discharged from the medical-surgical service were obtained from hospital administrative databases. Facilitywide acquisition and/or return costs and quantities for all systemic antimicrobial agents (antibacterial, antifungal and antiviral) were recorded monthly from pharmacy records for the baseline and intervention periods. In addition, administered quantities of antimicrobials were recorded monthly for each patient care location of the medical-surgical service and defined daily doses (DDDs; World Health Organization Center for Drug Statistics Methodology) were calculated. Antimicrobial acquisition costs were estimated by multiplying administered antimicrobial unit quantities by the 2009-2010 facility average acquisition price per unit. Incident healthcarefacility-onset CDI cases were scored and incidence rates calculated per 10,000 patient-days according to the
NHSN multidrug-resistant organism and CDI module protocol laboratory-identified event methodology [15].

\section{Statistical analysis}

Patient characteristics, antimicrobial use and cost, and CDI rates were compared during baseline and intervention periods. The Mann-Whitney $U$ test was used for continuous variable distributions, and the ${ }_{X}^{2}$ test was used for categorical variables. All reported $P$ values were twotailed with $P$ less than .05 as the level of significance. Statistical calculations were performed using GraphPad Prism, version 5.04.

\section{Results}

\section{Patient characteristics}

There were 1,422 admissions and 5,572 patient-days on the medical-surgical service during the 8-month baseline period and 3,076 admissions and 11,109 patient-days during the 16-month intervention period. Patient characteristics of the medical-surgical service are summarized in Table 1. Primary diagnoses were similar between the two periods except for diseases of the circulatory system $(P=.027)$. The mean monthly, facility-wide Medicare Case Mix Index increased from 1.4 to 1.6, or by $14 \%(P=.005)$.

There were 367 audits of 333 unique inpatient records during the stewardship period (Table 2). At the time of audit, 349 (95\%) patients had been prescribed antimicrobial therapy for three or more consecutive days, 168 
Table 1 Patient characteristics during baseline and intervention periods of an antimicrobial stewardship program

\begin{tabular}{|c|c|c|c|}
\hline Variable & Baseline & Intervention & $P^{a}$ \\
\hline Discharges, no. & 1409 & 3054 & \\
\hline Age, mean (SD), years & $57.4(18.6)$ & $57.4(18.7)$ & .933 \\
\hline Male sex & $628(44.6)$ & $1304(42.7)$ & .241 \\
\hline \multicolumn{4}{|l|}{ Race } \\
\hline Asian & $93(6.6)$ & $197(6.5)$ & .850 \\
\hline Black & $185(13.1)$ & $463(15.2)$ & .074 \\
\hline Hispanic & $180(12.8)$ & $377(12.3)$ & .686 \\
\hline White & $940(66.7)$ & $1971(64.5)$ & .156 \\
\hline \multicolumn{4}{|l|}{ Health insurance } \\
\hline Commercial, HMO, PPO & $627(44.5)$ & $1274(41.7)$ & .084 \\
\hline Medicare, Managed Care Medicare & $639(45.4)$ & $1382(45.3)$ & .951 \\
\hline Medicaid, Managed Care Medicaid & $37(2.6)$ & $95(3.1)$ & .374 \\
\hline ALOS, mean (SD), days & $3.9(0.3)$ & $3.6(0.3)$ & .118 \\
\hline \multicolumn{4}{|l|}{ Primary ICD-9 diagnosis code(s) } \\
\hline Circulatory & $314(22.3)$ & $593(19.4)$ & .027 \\
\hline Diabetes mellitus & $19(1.3)$ & $48(1.6)$ & .569 \\
\hline Digestive & $202(14.3)$ & $468(15.3)$ & .391 \\
\hline Genitourinary & $182(12.9)$ & $454(14.9)$ & .083 \\
\hline Infectious and parasitic & $57(4.0)$ & $110(3.6)$ & .468 \\
\hline Musculoskeletal and connective tissue & $61(4.3)$ & $160(5.2)$ & .193 \\
\hline Neoplasms & $144(10.2)$ & $293(9.6)$ & .513 \\
\hline Respiratory & $124(8.8)$ & $276(9.0)$ & .797 \\
\hline Skin and subcutaneous tissue & $43(3.1)$ & $81(2.7)$ & .450 \\
\hline
\end{tabular}

NOTE:

Data are no. (\%) of discharged medical-surgical service patients, unless otherwise indicated.

a - The Mann-Whitney $U$ test was used to compare continuous data, and the $X^{2}$ test was used to compare categorical data.

Abbreviations: ALOS, average length of stay; $\mathrm{HMO}$, health maintenance organization; ICD-9, International Classification of Diseases, $9^{\text {th }}$ Revision; PPO, preferred provider organization; SD, standard deviation.

(46\%) were receiving more than one agent, 206 (56\%) were receiving fluoroquinolones (levofloxacin) and 13 (4\%) were receiving two agents with anaerobic activity. Suspected or confirmed pulmonary infection was a rationale for antimicrobial therapy in 173 (47\%) audits and the most common indication for prescribed antimicrobial therapy.

Characteristics of AST recommendations are summarized in Table 3. The AST advised no change to the prescribed antimicrobial regimen for 54 audits and made one or more recommendation(s) to change the prescribed antimicrobial regimen (or consult ID) for 313 audits during the stewardship period. Physicians implemented recommendation(s) from each of 234 (75\%) audits, including from 85 of 115 for which discontinuation of all antimicrobial therapy was recommended.
Table 2 Characteristics of antimicrobial prescription regimens from 367 audits of 333 unique patient medical records

\begin{tabular}{|c|c|}
\hline Variable & $\begin{array}{l}\text { No. (\%) } \\
\text { of Audits }\end{array}$ \\
\hline Antimicrobial therapy $>2$ inpatient days & $349(95.1)$ \\
\hline \multicolumn{2}{|l|}{ Antimicrobial agent number } \\
\hline 1 & $199(54.2)$ \\
\hline$>1$ & $168(45.8)$ \\
\hline Parenteral agent(s) part of regimen & $336(91.6)$ \\
\hline Fluoroquinolone ${ }^{a}$ part of regimen & $206(56.1)$ \\
\hline Two anti-anaerobic agents ${ }^{\mathrm{b}}$ & $13(3.5)$ \\
\hline \multicolumn{2}{|l|}{ Antimicrobial indications ${ }^{c}$} \\
\hline Pulmonary & $173(47.1)$ \\
\hline Urinary & $90(24.5)$ \\
\hline Intra-abdominal & $88(24.0)$ \\
\hline Skin and soft tissue & $46(12.5)$ \\
\hline Other & $25(6.8)$ \\
\hline
\end{tabular}

\section{Antimicrobial use and cost, and CDI}

There was a $22 \%$ reduction in mean monthly use of all antimicrobial agents per 100 admissions $(P=.006)$ and a $32 \%$ reduction in cost per admission $(P=.013)$ in comparison to the baseline period (Table 4). Antimicrobial use and cost were also calculated per patient-day. There was a $16 \%$ reduction in mean monthly antimicrobial use per 1000 patient-days $(P=.013)$ and a 25\% reduction in cost per patient-day $(P=.022)$. There were statistically significant reductions from baseline in the use of antipseudomonal carbapenems (imipenem and meropenem), clindamycin, levofloxacin, linezolid, trimethroprim-sulfamethoxazole, antibacterials and antifungals using either metric denominator; there was a statistically significant increase from baseline in the use of cefazolin.

The mean monthly, incident healthcare-facility-onset CDI incidence rate for the medical-surgical service was 3.7 during the baseline period and 9.2 during the intervention period $(P=.232)$.

\section{Discussion}

In summary, we report the implementation of an ASP at a 100-bed community hospital employing a core strategy of post-prescriptive medical record audits and nonbinding AST recommendations with significant reductions in antimicrobial use and cost.

Respondents to a 2009 survey of the IDSA Emerging Infections Network on programmatic strategies and 
Table 3 Characteristics of 313 AST audits with one or more recommendations

\begin{tabular}{|c|c|c|c|}
\hline $\begin{array}{l}\text { Recommendation } \\
\text { category }\end{array}$ & $\begin{array}{l}\text { Number } \\
\text { of audits }\end{array}$ & $\begin{array}{l}\text { Implemented } \\
\text { recommendations }\end{array}$ & $\begin{array}{l}\text { Implementation } \\
\text { rate }(\%)\end{array}$ \\
\hline All & 313 & 234 & 75 \\
\hline $\begin{array}{l}\text { Discontinue all } \\
\text { agent(s) }\end{array}$ & 115 & 85 & 74 \\
\hline De-escalate ${ }^{a}$ & 65 & 53 & 82 \\
\hline Limit duration ${ }^{b}$ & 21 & 13 & 62 \\
\hline $\begin{array}{l}\text { Consult infectious } \\
\text { diseases }\end{array}$ & 19 & 16 & 84 \\
\hline Optimize dose & 14 & 7 & 50 \\
\hline Broaden $^{c}$ & 5 & 3 & 60 \\
\hline $\begin{array}{l}\text { Convert parenteral } \\
\text { to oral }\end{array}$ & 3 & 3 & 100 \\
\hline $\begin{array}{l}\text { More than } 1 \\
\text { category }\end{array}$ & 71 & 54 & 76 \\
\hline \multicolumn{4}{|c|}{$\begin{array}{l}\text { NOTE: } \\
\text { a - Discontinuation of one or more agent(s) and/or substitution of alternate } \\
\text { agent(s) with decreased spectrum of activity. } \\
\text { b - Limit duration for same agent(s) at same dose, route and schedule. } \\
\text { c - Addition of one or more agent(s) and/or substitution of alternate agent(s) } \\
\text { with increased spectrum of activity. } \\
\text { d - Parenteral to oral conversion(s) of same agent(s) at same dose and } \\
\text { schedule. }\end{array}$} \\
\hline
\end{tabular}

barriers for ASP implementation, reported that $61 \%$ of their hospitals had an ASP and that $12 \%$ were planning to initiate one [12]. However, respondents from hospitals with less than 200 beds reported that only $44 \%$ of their hospitals had an existing ASP; even so, the survey was thought to "likely overestimate the dissemination of ASPs". Lack of funding and/or personnel were considered to be primary barriers to ASP implementation.

Additional challenges in the implementation of our program included data management and the creation of data management tools, program documents, procedures, and reports as well as educational materials for the medical staff.

Although other reports have been published about ASPs implemented in community hospitals $[16,17]$, only one has previously described an ASP at a facility in the United States with less than 200 beds. In 2003, LaRocco described an antibiotic support team developed at a 120bed facility in Louisiana [14]. Concurrent chart review was performed three days per week focusing on multiple, prolonged and high-cost antibiotic therapies. There was a $19 \%$ savings on antibiotic costs per patient-day over a 12-month intervention period. We employed a similar core strategy but with a 2-person ID physician/clinical pharmacist team that audited medical records two days per week. We limited our audits to patients on the medical-surgical service, as others have reported [18], and demonstrated a $25 \%$ cost reduction per patient-day $(P=.022)$. LaRocco did not report use metrics; there may be other unknown differences in the setting, patient characteristics, interventions, and data analysis limiting any further comparisons between these two programs.

The generation of recommended antimicrobial use metrics was among our greatest challenges. We selected DDD as recommended by the IDSA/SHEA stewardship guidelines [9]. Days of therapy were not available from pharmacy records. These same stewardship guidelines are silent on the recommendation for a metric denominator(s); we calculated both DDD per admission and per patient day, as recommended by others [19-21].

There were few differences in patient characteristics between the baseline and intervention periods. The facility-wide Medicare Case Mix Index increased significantly during the intervention period; however it is unclear how this may be related to antimicrobial use on the medical-surgical service.

Severe sepsis order sets and a parenteral to oral conversion protocol were implemented during the intervention period. The order sets did not limit duration of antimicrobial therapy and the parenteral to oral conversion protocol included only five antimicrobial agents eligible for substitution at the same dose and frequency. Nevertheless, we cannot exclude the possibility that these additional interventions may have had an impact on antimicrobial use and cost.

Similar to other recent reports from the United States, we observed more than one antimicrobial agent was prescribed for $46 \%$ of audited records. In a retrospective study of adult, nonpsychiatric inpatients prescribed two or more consecutive days of antibiotic therapy at a tertiary care hospital in New York, two or more antibiotic agents were employed for $63 \%$ percent of 10,154 hospitalizations [22]. In an observational study of adult inpatients prescribed fluoroquinolones at a tertiary care hospital in Ohio, 56\% of 227 regimens combined fluoroquinolones with antibiotic agents from other classes [23].

We observed a pulmonary source of infection was an indication for antimicrobial therapy in $47 \%$ of audited records. In addition, a fluoroquinolone, levofloxacin, was prescribed in 56\% of audited records and accounted for approximately $30 \%$ of overall antibiotic consumption on the medical-surgical service during both the baseline and intervention periods. We speculate that these findings may in part be related to antibiotic choices in a pneumonia order set designed to align with the Centers for Medicaid and Medicare Services National Inpatient Quality Measure PN-6, "Initial Antibiotic Selection for Community-Acquired Pneumonia (CAP) in Immunocompetent Patients" [24].

Discontinuation of all antimicrobial therapy accounted for $36 \%(85 / 234)$ of audited records with implemented recommendations. The observed reductions in the use of levofloxacin, and of antimicrobials overall, would likely not have been realized if we had chosen a core 
Table 4 Antimicrobial use and cost during baseline and intervention periods of an antimicrobial stewardship program

\begin{tabular}{|c|c|c|c|c|c|c|}
\hline \multirow[t]{2}{*}{ Variable } & \multicolumn{3}{|c|}{ DDD per 100 Admissions } & \multicolumn{3}{|c|}{ DDD per 1000 Patient-days } \\
\hline & Baseline & Intervention & $P^{\mathrm{a}}$ & Baseline & Intervention & $P^{\mathrm{a}}$ \\
\hline \multicolumn{7}{|l|}{ By category } \\
\hline Antibacterials & 401.8 & 318.9 & .009 & 1028 & 878.7 & .011 \\
\hline Antifungals & 23.1 & 13.2 & .035 & 59.1 & 36.5 & .047 \\
\hline Antivirals & 7.5 & 5.2 & .375 & 21.3 & 14.2 & .257 \\
\hline All agents & 432.4 & 337.3 & .006 & 1109 & 929.4 & .013 \\
\hline \multicolumn{7}{|l|}{ By selected class } \\
\hline Antipseudomonal carbapenems ${ }^{b}$ & 13.8 & 4.4 & .047 & 35.0 & 12.4 & .047 \\
\hline Cephalosporins & 42.2 & 49.2 & .188 & 108.7 & 135.7 & .030 \\
\hline Echinocandins & 4.7 & 3.2 & .975 & 11.3 & 8.7 & .924 \\
\hline Fluoroquinolones & 123.1 & 100.8 & .011 & 314.2 & 279.2 & .071 \\
\hline \multicolumn{7}{|l|}{ By selected agent } \\
\hline Ampicillin-sulbactam & 17.6 & 16.1 & .830 & 46.8 & 43.9 & .878 \\
\hline Cefazolin & 20.8 & 26.9 & .013 & 53.7 & 74.9 & .004 \\
\hline Ceftriaxone & 13.2 & 14.5 & .603 & 34.3 & 39.8 & .312 \\
\hline Cefepime & 0.5 & 1.9 & .217 & 1.3 & 5.0 & .171 \\
\hline Clindamycin & 22.5 & 12.7 & .004 & 57.4 & 34.8 & .009 \\
\hline Daptomycin & 2.0 & 0.7 & .913 & 5.1 & 2.1 & .855 \\
\hline Ertapenem & 14.2 & 12.4 & .878 & 35.6 & 34.0 & .830 \\
\hline Fluconazole & 17.6 & 9.8 & .105 & 46.1 & 27.2 & .284 \\
\hline Levofloxacin & 122.0 & 97.1 & .006 & 311.2 & 269.2 & .030 \\
\hline Linezolid & 4.5 & 0.5 & .017 & 11.3 & 1.6 & .020 \\
\hline Metronidazole & 21.7 & 16.9 & .105 & 55.5 & 46.1 & .257 \\
\hline Nafcillin & 3.0 & 0.3 & .370 & 7.3 & 0.9 & .370 \\
\hline Piperacillin-tazobactam & 32.0 & 30.8 & .783 & 80.7 & 84.7 & .736 \\
\hline Tigecycline & 0.2 & 0.2 & .702 & 0.5 & 0.4 & .702 \\
\hline Trimethoprim-sulfamethoxazole & 25.6 & 3.9 & .016 & 65.0 & 10.8 & .017 \\
\hline \multirow[t]{3}{*}{ Vancomycin } & 44.8 & 42.7 & .783 & 115.2 & 116.2 & .976 \\
\hline & \multicolumn{3}{|c|}{ US\$ per Admission } & \multicolumn{3}{|c|}{ US\$ per Patient-day } \\
\hline & Baseline & Intervention & $P^{a}$ & Baseline & Intervention & $p^{a}$ \\
\hline \multicolumn{7}{|l|}{ By category } \\
\hline Antibacterials & 79.8 & 54.7 & .025 & 20.2 & 15.1 & .040 \\
\hline All agents & 87.0 & 59.4 & .013 & 22.0 & 16.4 & .022 \\
\hline
\end{tabular}

\section{NOTE:}

Data are means of monthly medical-surgical service antimicrobial use and cost.

a - The Mann-Whitney $U$ test.

b - Imipenem and meropenem (doripenem was not used). A formulary change (and auto-substitution policy) from meropenem to imipenem was implemented during the intervention period.

Abbreviations: DDD, defined daily doses (World Health Organization Center for Drug Statistics Methodology).

strategy of antibiotic restriction focused on one or more high cost agent(s). Although inexpensive compared to other antimicrobials and available for oral administration, fluoroquinolones have been associated with both methicillin-resistant $S$. aureus and CDI in hospitals $[25,26]$. Receipt of fluoroquinolones and all antibiotics have also been shown to be independent risk factors for carbapenem-resistant $K$. pneumoniae acquisition among hospitalized adults [27].

Measurement of changes in antimicrobial resistance patterns associated with antimicrobial stewardship has been recommended as a potential outcome measure for ASPs [9]. In the current report, small numbers of unique clinical isolates precluded a meaningful assessment of 
the program's impact on antibiotic resistance. Likewise, a switch from enzyme immunoassay detection of toxins to a polymerase chain reaction assay in month 3 (November 2009) of the 16-month ASP intervention period likely confounded comparison of healthcare-facility-onset CDI rates. (A recent report demonstrated significant increases and an approximate doubling of the prevalence of positive laboratory tests for CDI and the CDI rate after a similar switch in detection methods [28]). Also, factors other than antibiotic use may affect CDI, and we were unable to draw any conclusions about the statistically unchanged CDI rates between the periods [29].

\section{Conclusions}

In conclusion, an effective ASP was implemented at a 100-bed community hospital. Importantly, there were significant reductions in overall antimicrobial use. More focus is needed on antimicrobial stewardship strategies, measures and resources in this healthcare setting.

\section{Competing interests}

The authors declare that they have no competing interests.

\section{Authors' contributions}

DS, PP, AN, and FC shared in program conception and design. DS served as the physician champion for the medical staff. PP developed the data management tool and performed data analysis. DS, AN, and FC performed medical record audits. DS and PP drafted the initial manuscript. All authors have read and approved the final manuscript.

\section{Previous presentations}

Presented in part: $21^{\text {st }}$ Annual Scientific Meeting of the Society for Healthcare Epidemiology of America, Dallas, TX, 1-4 April 2011 [Abstract 77].

\section{Acknowledgements}

We thank Dr. Bob Bolton for assistance with medical record audits and Dr. Linda Hynan for her helpful statistical review of the manuscript.

\section{Author details}

${ }^{1}$ Dallas ID Associates, Dallas, Texas, USA. ${ }^{2}$ Medical City Dallas Hospital, Dallas, Texas, USA. ${ }^{3}$ Medical Center of McKinney, McKinney, Texas, USA.

Received: 22 May 2012 Accepted: 4 October 2012

Published: 9 October 2012

\section{References}

1. National Center for Health Statistics: Health, United States, 2010; With Special Feature on Death and Dying. Hyattsville, MD; 2010.

2. Dudeck MA, Horan TC, Peterson KD, Allen-Bridson K, Morrell G, Pollock DA Edwards JR: National Healthcare Safety Network (NHSN) report, data summary for 2010, device-associated module. Am J Infect Control 2011 , 39:798-816.

3. Klevens RM, Edwards JR, Gaynes RP: National Nosocomial Infections Surveillance System. The impact of antimicrobial-resistant, healthcareassociated infections on mortality in the United States. Clin Infect Dis 2008, 47:927-930

4. Jernigan JA: Overview of antimicrobial resistance - antibacterial resistance. FDA Public Meeting: Antibacterial Resistance and Diagnostic Device and Drug Development Research for Bacterial Diseases; Public Workshop, Silver Spring, MD, 26-27 July 2010. http://www.fda.gov/downloads/Drugs/NewsEvents/ UCM226072.pdf.

5. Campbell RJ, Giljahn L, Machesky A, Cibulskas-White K, Lane LM, Porter K, Paulson JO, Smith FW, McDonald LC: Clostridium difficile infection in Ohio hospitals and nursing homes during 2006. Infect Control Hosp Epidemiol 2009, 30:526-533.
6. Bartlett JG: A call to arms: the imperative for antimicrobial stewardship. Clin Infect Dis 2011, 53(Suppl 1):4-7.

7. Septimus EJ, Owens RC Jr: Need and potential of antimicrobial stewardship in community hospitals. Clin Infect Dis 2011, 53(Suppl 1):8-14.

8. Ohl CA, Dodds Ashley ES: Antimicrobial stewardship programs in community hospitals: the evidence base and case studies. Clin Infect Dis 2011, 53(Suppl 1):23-28.

9. Dellit TH, Owens RC, McGowan JE Jr, Gerding DN, Weinstein RA, Burke JP, Huskins WC, Paterson DL, Fishman NO, Carpenter CF, Brennan PJ, Billeter M, Hooton TM, Infectious Diseases Society of America, Society for Healthcare Epidemiology of America: Infectious Diseases Society of America and the Society for Healthcare Epidemiology of America guidelines for developing an institutional program to enhance antimicrobial stewardship. Clin Infect Dis 2007, 44:159-177.

10. Barlam TF, DiVall M: Antibiotic-stewardship practices at top academic centers throughout the United States and at hospitals throughout Massachusetts. Infect Control Hosp Epidemiol 2006, 27:695-703.

11. Trivedi $K$, Rosenberg J: The state of antimicrobial stewardship programs in California. 21st Annual Scientific Meeting of the Society for Healthcare Epidemiology of America, Dallas, TX 1-4 April 2011 [Abstract 376]. http://shea. confex.com/shea/2011/webprogram/Paper4907.html.

12. Johannsson B, Beekman SE, Srinivasan A, Hersh AL, Laxminarayan R, Polgreen PM: Improving antimicrobial stewardship: the evolution of programmatic strategies and barriers. Infect Control Hosp Epidemiol 2011, 32:367-374.

13. Kuntz JL, Cavanaugh JE, Becker LK: Clostridium difficile-associated disease in patients in a small rural hospital. Infect Control Hosp Epidemiol 2007, 28:1236-1239.

14. LaRocco A Jr: Concurrent antibiotic review programs - a role for infectious diseases specialists at small community hospitals. Clin Infect Dis 2003, 37:742-743.

15. National Healthcare Safety Network (NHSN): Multidrug-Resistant Organism \& Clostridium difficile Infection (MDRO/CDI) Module Protocol. http://www.cdc gov/nhsn/PDFs/pscManual/12pscMDRO_CDADcurrent.pdf.

16. Carling P, Fung T, Killion A, Terrin N, Barza M: Favorable impact of a multidisciplinary antibiotic management program conducted during 7 years. Infect Control Hosp Epidemiol 2003, 24:699-706.

17. Ruttiman S, Keck B, Hartmeier C, Maetzel A, Bucher HC: Long-term antibiotic cost savings from a comprehensive intervention program in a medical department of a university-affiliated teaching hospital. Clin Infect Dis 2004, 38:348-356.

18. Fraser GL, Stogsdill P, Dickens JD Jr, Wennberg DE, Smith RP, Prato S: Antibiotic optimization. An evaluation of patient safety and economic outcomes. Arch Int Med 1997, 157:1689-1694.

19. Filius PMG, Liem TB, van der Linden PD, Janknegt $R$, Natsch S, Vulto AG, Verbrugh $\mathrm{HA}$ : An additional measure for quantifying antibiotic use in hospitals. J Antimicrob Chemother 2005, 55:805-808.

20. Ansari F, Molana H, Goossens H, Davey P: Development of standardized methods for analysis of changes in antibacterial use in hospitals from 18 European countries: the European Surveillance of Antimicrobial Consumption (ESAC) longitudinal survey, 2000-06. J Antimicrob Chemother 2010, 65:2686-2691.

21. Jacob JT, Gaynes RP: Emerging trends in antibiotic use in US hospitals: quality, quantification and stewardship. Expert Rev Anti Infect Ther 2010, 8:893-902

22. Stevens V, Dumyati G, Fine LS, Fisher SG, van Winjgaarden E: Cumulative antibiotic exposures over time and the risk of Clostridium difficile infection. Clin Infect Dis 2011, 53:42-48.

23. Werner NL, Hecker MT, Sethi AK, Donskey CJ: Unnecessary use of fluoroquinolone antibiotics in hospitalized patients. BMC Infect Dis 2011, 11:187.

24. Centers for Medicaid and Medicare Services and The Joint Commission Specifications Manual for National Hospital Inpatient Quality Measures. http:// www.jointcommision.org/specifications_manual_for_national_hospital_ inpatient_quality_measures.aspx.

25. Pepin J, Saheb N, Coulombe MA, Alary ME, Corriveau MP, Authier S, Leblanc M, Rivard G, Bettez M, Primeau V, Nguyen M, Jacob CE, Lanthier L: Emergence of fluoroquinolones as the predominant risk factor for Clostridium difficile-associated diarrhea: a cohort study during an epidemic in Quebec. Clin Infect Dis 2005, 41:1254-1260. 
26. MacDougall C, Powell JP, Johnson CK, Edmond MB, Polk RE: Hospital and community fluoroquinolone use and resistance in Staphylococcus aureus and Escherichia coli in 17 US hospitals. Clin Infect Dis 2005, 41:435-440.

27. Schwaber MJ, Klarfeld-Lidji S, Navon-Venezia S, Schwartz D, Leavitt A, Carmeli Y: Predictors of carbapenem-resistant Klebsiella pneumoniae acquisition among hospitalized adults and effect of acquisition on mortality. Antimicrob Agents Chemother 2008, 52:1028-1033.

28. Fong KS, Fatica C, Hall G, Procop G, Schindler S, Gordon SM, Fraser TG: Impact of PCR testing for Clostridium difficile on incident rates and potential on public reporting: is the playing field level? Infect Control Hosp Epidemiol 2011, 32:932-933.

29. Cohen SH, Gerding DN, Johnson S, Kelly CP, Loo VG, McDonald LC, Pepin J, Wilcox MH, Society for Healthcare Epidemiology of America, Infectious Diseases Society of America: Clinical practice guidelines for Clostridium difficile infection in adults: 2010 update by the Society for Healthcare Epidemiology of America (SHEA) and the Infectious Diseases Society of America (IDSA). Infect Control Hosp Epidemiol 2010, 31:431-455.

doi:10.1186/2047-2994-1-32

Cite this article as: Storey et al: Implementation of an antimicrobial stewardship program on the medical-surgical service of a 100-bed community hospital. Antimicrobial Resistance and Infection Control 2012 1:32.

\section{Submit your next manuscript to BioMed Central and take full advantage of:}

- Convenient online submission

- Thorough peer review

- No space constraints or color figure charges

- Immediate publication on acceptance

- Inclusion in PubMed, CAS, Scopus and Google Scholar

- Research which is freely available for redistribution 